\title{
QCD phase diagram with a background magnetic field in an improved soft-wall AdS/QCD model
}

\author{
Zhen Fang ${ }^{1, a}$, Ying-Ying $\mathbf{L i}^{1, b}$, Yue-Liang $\mathbf{W u}^{2,3, \mathrm{c}}$ \\ ${ }^{1}$ Department of Applied Physics, School of Physics and Electronics, Hunan University, Changsha 410082, People's Republic of China \\ ${ }^{2}$ CAS Key Laboratory of Theoretical Physics, Institute of Theoretical Physics, Chinese Academy of Sciences, Beijing 100190, People's Republic \\ of China \\ ${ }^{3}$ International Centre for Theoretical Physics Asia-Pacific (ICTP-AP), University of Chinese Academy of Sciences, Beijing 100049, People's \\ Republic of China
}

Received: 13 January 2021 / Accepted: 7 June 2021 / Published online: 25 June 2021

(C) The Author(s) 2021

\begin{abstract}
We studied the magnetic effects on the chiral transition and the melting properties of vector and axialvector mesons in the improved soft-wall AdS/QCD model under a charged magnetic background, which is solved perturbatively from an Einstein-Maxwell system with a negative cosmological constant. The phase diagrams for both chiral transition and meson melting have been obtained. We show that the inverse magnetic catalysis emerged naturally in the improved soft-wall model. We also find that the magnetic field can induce meson melting, at least for the vector and axial-vector mesons, in our holographic setup.
\end{abstract}

\section{Introduction}

The QCD phase diagram in the presence of a magnetic field has been studied extensively over the past decades [1]. There exists various environments involving both the strong interaction and the magnetic field. For instance, a strong magnetic field around $\sqrt{e B} \sim 1.5 \mathrm{GeV}$ may be produced during the period of electroweak phase transition [2], and a magnetic field around $\sqrt{e B} \sim \Lambda_{\mathrm{QCD}}$ may also be generated in the noncentral heavy ion collisions at the Relativistic Heavy Ion Collider and the Large Hadron Collider [3]. The compact dense stars such as the magnetars can produce magnetic field of the order of $\sqrt{e B} \sim 1 \mathrm{MeV}$. Thus, it is worthwhile to figure out the new features of the QCD phase diagram when the magnetic field were added. In addition, it is intriguing and important to address such issues as the magnetization of the QCD vacuum.

\footnotetext{
a e-mail: zhenfang@hnu.edu.cn (corresponding author)

be-mail: lyying@hnu.edu.cn

c e-mail: ylwu@itp.ac.cn
}

A variety of approaches and models have been used to study the QCD phase diagram with a magnetic field. The lattice QCD can tackle these issues with no sign problem, unlike the case of finite chemical potential. In the study of the magnetic effects on the transition temperature, many works predicted a magnetic catalysis, i.e., the transition temperature increases with increasing magnetic field [4-7]. However, the lattice simulations have indicated that the critical temperature decreases with the increase of the magnetic field, which is termed inverse magnetic catalysis (IMC) $[8,9]$. This effect conforms with some earlier results obtained from chiral perturbation theory [10] or the bag model [11]. The (Polyakov-)Nambu-Jona-Lasinio models can also generate the IMC [12]. The lattice results further motivate the subsequent research of the QCD phase transition under a magnetic field. Some works indicated that a strong magnetic field can change the order of chiral phase transition [13], which is yet not favored by lattice simulations [8]. The mechanism of IMC has also been discussed in the previous studies, e.g., the IMC may originate from the magnetic inhibition [14], or may be induced by the spharelons [15].

In this work, we consider the related issues of the QCD phase diagram with magnetic field in the holographic framework originated from the anti-de Sitter/conformal field theory (AdS/CFT) correspondence, which is a holographic duality between a weakly coupled supergravity theory in $\mathrm{AdS}_{5}$ and a super-Yang-Mills theory on the boundary [16-19]. There has been a large amount of research in the so called AdS/QCD over the past two decades ranging from the lowenergy hadron physics [20-42] to the QCD thermodynamics with non-perturbative aspects dominated [43-78].

There are also many holographic studies for the magnetic effects of the QCD phase transition [79-87]. Although most holographic QCD models exhibit magnetic catalysis, there 
are some ones in which the inverse magnetic catalysis does manifest. It has been shown that the chiral phase transition in the Sakai-Sugimoto model has the property of magnetic catalysis [7]. While for the same model but in a limit of small separation of the flavor D8-branes, the inverse magnetic catalysis manifests at finite chemical potential [88]. The deconfining phase transition also shows inverse magnetic catalysis in the Sakai-Sugimoto model [83]. In Ref. [84], the author computed the deconfinement critical temperature under the influence of a background magnetic field within the hard-wall model and the holographic duals of flavored and unflavored $\mathcal{N}=4$ super Yang-Mills theories on $\mathbb{R}^{3} \times S^{1}$, and showed that the inverse magnetic catalysis happens for these cases when $B \ll T^{2}$. In addition, some other holographic QCD models have also been shown to exhibit inverse magnetic catalysis [88-90].

In Ref. [91], we proposed a simply improved soft-wall AdS/QCD model which is able to characterize the spontaneous chiral symmetry breaking and the linear confinement, and gives a consistent description for the light meson spectra and the chiral phase transition in the two-flavor case. In this work, we will give a further study on the QCD phase diagram in the improved soft-wall AdS/QCD model with a charged magnetic background, which can be solved perturbatively from an Einstein-Maxwell system [92]. The chiral transition under the influence of magnetic field will be investigated, and the melting properties of the vector and axialvector mesons in terms of the thermal spectral functions will also be studied. We obtain the phase diagram for both cases with the existence of magnetic field, and we find that the inverse magnetic catalysis manifests in both cases. There are also some earlier studies on the QCD phase diagram at finite $T, \mu$ and $B$ in terms of bottom-up holographic models [9395]. Although with different holographic setups and different variables addressed, all these models can generate the effect of inverse magnetic catalyses.

The paper is organized as follows. In Sect. 2, we give an outline of the simply improved soft-wall AdS/QCD model and introduce the charged magnetic background, which will be derived in Appendix. In Sect. 3, we investigate the magnetic and chemical potential effects on chiral transition and obtain the chiral phase diagram with the magnetic field added. In Sect. 4, we compute the spectra functions for the vector and axial-vector mesons, and extract the melting critical temperatures at each chemical potential and magnetic field. Then the phase diagram is also obtained. In Sect. 5, we summarize our work and give a conclusion.

\section{The improved soft-wall model with a charged magnetic background}

\subsection{The action of the model}

We first outline the simply improved soft-wall AdS/QCD model with two flavors, which can reproduce the light meson spectra matching with experiments [91]. The chiral transition obtained from this model has a crossover behavior at physical quark mass and becomes a second-order phase transition in the chiral limit, which is also consistent with the standard scenario of the QCD phase diagram in the two-flavor case.

The bulk action of this improved soft-wall model can be written as

$$
\begin{aligned}
S_{M}= & -\int d^{5} x \sqrt{-g} e^{-\Phi(z)} \operatorname{Tr}\left\{|D X|^{2}+m_{5}^{2}(z)|X|^{2}+\lambda|X|^{4}\right. \\
& \left.+\frac{1}{4 g_{5}^{2}}\left(F_{L}^{2}+F_{R}^{2}\right)\right\}
\end{aligned}
$$

with a bulk scalar field $X$ and the SU(2) chiral gauge fields $A_{L, R}^{M}$. The covariant derivative of the scalar field is $D^{M} X=\partial^{M} X-i A_{L}^{M} X+i X A_{R}^{M}$, and the gauge field strength is $F_{L, R}^{M N}=\partial^{M} A_{L, R}^{N}-\partial^{N} A_{L, R}^{M}-i\left[A_{L, R}^{M}, A_{L, R}^{N}\right]$. The gauge coupling constant can be fixed as $g_{5}^{2}=\frac{12 \pi^{2}}{N_{c}}$ with the color number $N_{c}=3$ [24]. The dilaton field takes the form $\Phi(z)=\mu_{g}^{2} z^{2}$ to reproduce the Regge behavior of the hadron spectra, and the mass of the bulk scalar field takes the form $m_{5}^{2}(z)=-3-\mu_{c}^{2} z^{2}$ in order to generate the mass split of the vector and axial-vector mesons [91].

\subsection{The bulk geometry with a magnetic field}

To study the effects of magnetic field on QCD phase transition at finite chemical potential, we need a charged magnetic black hole solution, which can be obtained by solving perturbatively the 5-dimensional (5D) Einstein-Maxwell system with an electric charge density $\rho$ and a constant magnetic field $B$ along the $x_{3}$ axis (see Appendix for details) [92]. As a preliminary attempt, we adopt such a perturbative solution in our work.

The metric ansatz for the charged magnetic black hole up to order $B^{2}$ reads

$$
\begin{aligned}
d s^{2}= & e^{2 A(z)}\left(-f(z) d t^{2}+\frac{d z^{2}}{f(z)}\right. \\
& \left.+e^{2 P(z)}\left(d x_{1}^{2}+d x_{2}^{2}\right)+e^{2 Q(z)} d x_{3}^{2}\right),
\end{aligned}
$$

where we take $A(z)=-\ln \frac{z}{L}$ that fixes the gauge of the metric form (the AdS radius is set to be $L=1$ ), and

$$
Q(z)=-2 P(z)=B^{2} W_{2}(1 / z),
$$




$$
\begin{aligned}
f(z)= & 1-\left(1+Q^{2}\right)\left(\frac{z}{z_{h}}\right)^{4}+Q^{2}\left(\frac{z}{z_{h}}\right)^{6} \\
& +\frac{2}{3} B^{2} z^{4} \ln \frac{z}{z_{h}}, \quad Q=q z_{h}^{3}
\end{aligned}
$$

with $q=\frac{\rho}{\sqrt{3}}$ being the charge of black hole and

$$
W_{2}(r)=\frac{4}{3} \int_{\infty}^{r} d r^{\prime} \frac{\ln \left(r^{\prime} / r_{h}\right)}{r^{\prime 3} U_{0}\left(r^{\prime}\right)}, \quad U_{0}(r)=r^{2}+\frac{q^{2}}{r^{4}}-\frac{M}{r^{2}} .
$$

The Hawking temperature is determined by

$$
T=\left|\frac{f^{\prime}\left(z_{h}\right)}{4 \pi}\right|=\frac{1}{\pi z_{h}}\left|1-\frac{2 \mu^{2} z_{h}^{2}}{3}-\frac{B^{2} z_{h}^{4}}{6}\right|,
$$

where the chemical potential $\mu$ is related to the charge $q$ of the black hole by

$\mu=\frac{\sqrt{3}}{2} q z_{h}^{2}=\frac{\sqrt{3}}{2} \frac{Q}{z_{h}}$.

Following Ref. [86], we take the physical magnetic field to be $\mathcal{B}=1.6 \frac{B}{L}$.

\section{Chiral transition in the charged magnetic background}

\subsection{The EOM of the scalar VEV and the boundary condition}

we shall consider the vacuum part of the model in order to study the chiral transition properties [24]. The chiral condensate is incorporated in the ultraviolet (UV) asymptotic form of the vacuum expectation value (VEV) of the bulk scalar field which is set to be $\langle X\rangle=\frac{\chi(z)}{2} I_{2}$, where $I_{2}$ denotes the $2 \times 2$ identity matrix. The action of the scalar VEV $\chi(z)$ can be obtained from the bulk action (1) as

$$
S_{\chi}=-\int d^{5} x \sqrt{-g} e^{-\Phi}\left(\frac{1}{2} g^{z z}\left(\partial_{z} \chi\right)^{2}+V(\chi)\right),
$$

where the potential of the scalar VEV takes the form

$$
V(\chi)=\operatorname{Tr}\left\{m_{5}^{2}\langle X\rangle^{2}+\lambda\langle X\rangle^{4}\right\}=\frac{m_{5}^{2}}{2} \chi^{2}+\frac{\lambda}{8} \chi^{4} .
$$

The equation of motion (EOM) of $\chi(z)$ can be derived from the variation of $S_{\chi}$ with respect to $\chi$,

$$
\chi^{\prime \prime}+\left(3 A^{\prime}-\Phi^{\prime}+\frac{f^{\prime}}{f}\right) \chi^{\prime}-\frac{e^{2 A}}{f} \partial_{\chi} V(\chi)=0 .
$$

By the dictionary of AdS/CFT [24], the UV asymptotic form of $\chi(z)$ can be obtained from Eq. (9) as

$$
\begin{aligned}
\chi(z \sim 0)= & m_{q} \zeta z+\frac{\sigma}{\zeta} z^{3} \\
& +\left(m_{q} \zeta \mu_{g}^{2}-\frac{1}{2} m_{q} \zeta \mu_{c}^{2}+\frac{1}{4} m_{q}^{3} \zeta^{3} \lambda\right) z^{3} \ln z+\cdots,
\end{aligned}
$$

where $m_{q}$ denotes the current quark mass and $\sigma$ denotes the chiral condensate, and $\zeta=\frac{\sqrt{N_{c}}}{2 \pi}$ is a normalization constant which is fixed by matching the QCD result of the $N_{c}$ scaling behavior of $m_{q}$ and $\sigma$ with the holographic calculation [28]. The regularity of Eq. (9) at the horizon $z_{h}$ imposes the natural IR boundary condition:

$\left[f^{\prime} \chi^{\prime}-e^{2 A} \partial_{\chi} V(\chi)\right]_{z=z_{h}}=0$.

With the above boundary condition, we can solve Eq. (9) numerically to obtain the profile of the scalar VEV $\chi(z)$ whereby the chiral condensate $\sigma$ can be extracted as a function of the temperature $T$, the chemical potential $\mu$ and the magnetic field $\mathcal{B}$. Then we can investigate the properties of chiral transition and the chiral phase diagram.

\subsection{Chiral transition and chiral phase diagram}

To implement the numerical calculation, we take the values of the model parameters as $\lambda=80, \mu_{g}=440 \mathrm{MeV}, \mu_{c}=$ $1450 \mathrm{MeV}$ and $m_{q}=3.22 \mathrm{MeV}$, which have been shown to reproduce the consistent mass spectra for the pseudoscalar, vector and axial-vector mesons, and also realize the correct chiral transition behaviors [91]. We then investigate the chiral transition properties under the influence of magnetic field at finite chemical potential.

The numerical results are presented in Fig. 1. The $\sigma(T)$ curves at $\mu=0,0.5,0.8 \mathrm{GeV}$ and $\mathcal{B}=0,0.5,0.8 \mathrm{GeV}^{2}$ are plotted on the left panel of Fig. 1, from which we can see obviously the crossover behavior of the chiral transition. The transition temperature $T_{\chi}$ can be defined as the extreme point of $\partial \sigma / \partial T$. We show the $T$-dependent curves of $\partial \sigma / \partial T$ at each value of $\mu$ and $\mathcal{B}$ on the right panel of Fig. 1, from which we find that both the chiral condensate $\sigma$ and the chiral transition temperature $T_{\chi}$ decrease with the increase of $\mu$ at fixed $\mathcal{B}$, while the variations of $\sigma$ and $T_{\chi}$ with $\mathcal{B}$ at fixed $\mu$ exhibit more complicated behaviors.

For the case of $\mu=0$, we can see obviously that the chiral condensate $\sigma$ decreases with the increase of $\mathcal{B}$ at higher temperature, while the behavior inverses at lower temperature. Thus for the chiral condensate $\sigma$, our model correctly displays magnetic catalysis at low temperatures and inverse magnetic catalysis at higher temperatures, which is consistent with the lattice results, although the magnetic catalysis at low temperatures looks much smaller than the lattice data $[8,9]$. 

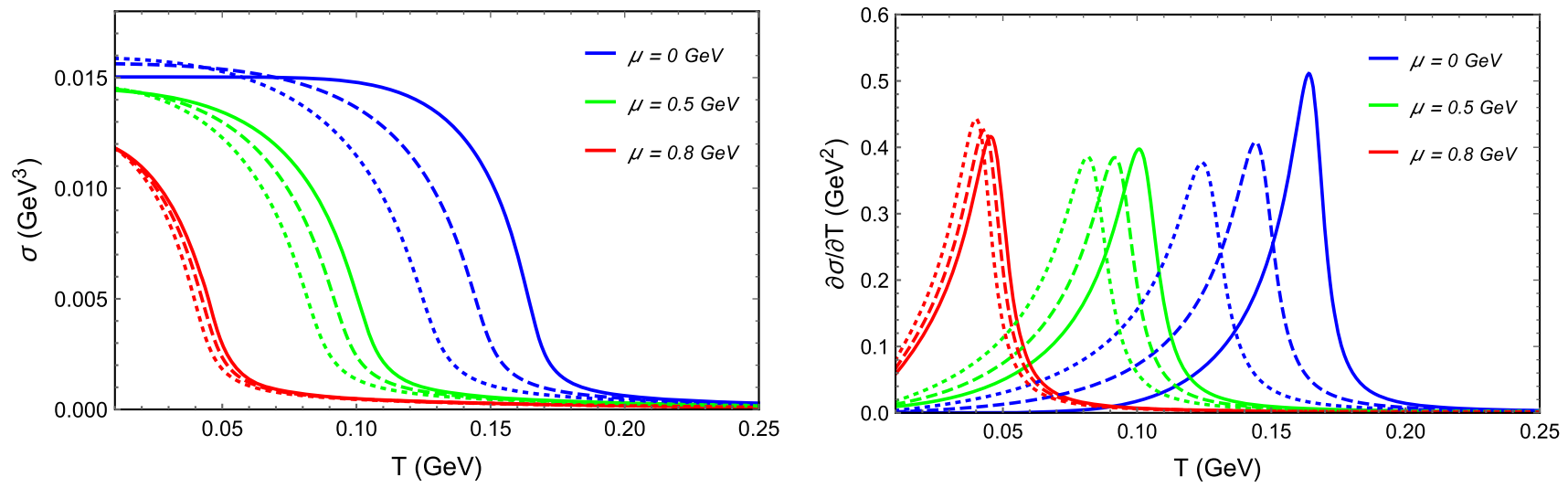

Fig. 1 The chiral transition behaviors (left panel) and the variations of $\partial \sigma / \partial T$ (right panel) with different $\mu$ and $\mathcal{B}$. The solid, dashed and dotted curves denote the cases with $\mathcal{B}=0,0.5,0.8 \mathrm{GeV}^{2}$ respectively, while the blue, green and red colors denote the cases with $\mu=0,0.5,0.8 \mathrm{GeV}$ respectively
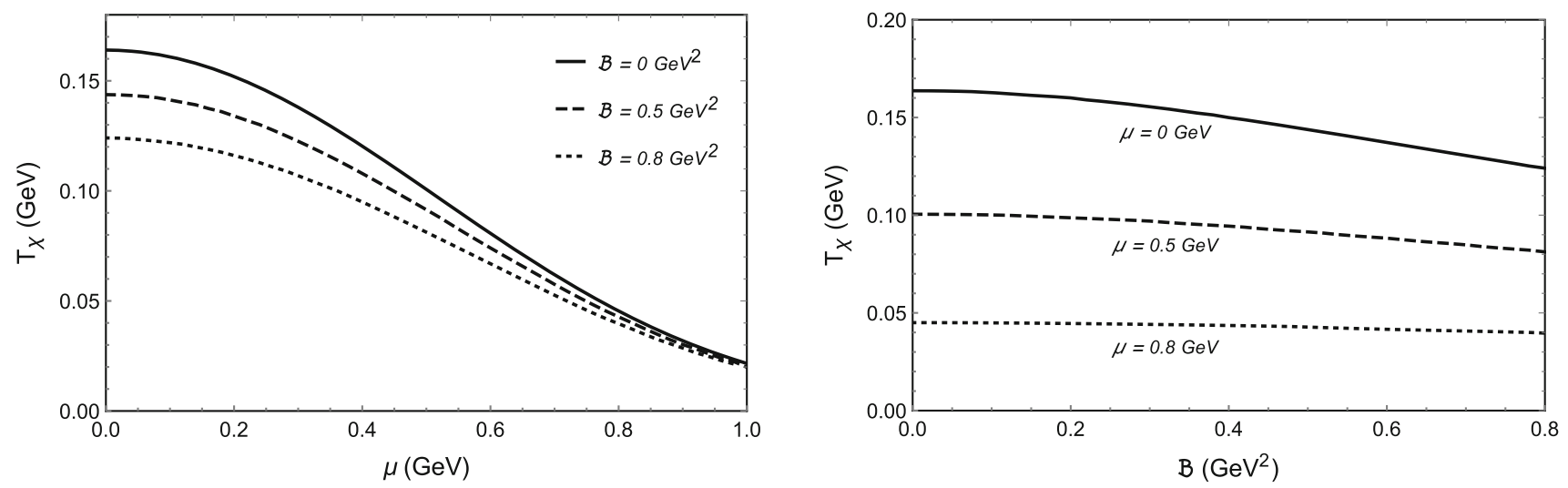

Fig. 2 The $\mu-T_{\chi}$ phase diagram at $\mathcal{B}=0,0.5,0.8 \mathrm{GeV}^{2}$ (left panel) and the $\mathcal{B}-T_{\chi}$ phase diagram at $\mu=0,0.5,0.8 \mathrm{GeV}$ (right panel)

This effect is further weakened with the increase of $\mu$. Moreover, from the right panel of Fig. 1, we can see clearly the existence of inverse magnetic catalysis for $T_{\chi}$ in our model, which is also weakened with the increase of $\mu$.

To make a better comparison, we show in Fig. 2 the $\mu-T_{\chi}$ phase diagram at $\mathcal{B}=0,0.5,0.8 \mathrm{GeV}^{2}$ and the $\mathcal{B}-T_{\chi}$ phase diagram at $\mu=0,0.5,0.8 \mathrm{GeV}$, from which we can see that the influence of $\mathcal{B}$ on $T_{\chi}$ is relatively weaker than that of $\mu$ on $T_{\chi}$. From the left panel of Fig. 2, we find that the chiral transition temperature $T_{\chi}$ descends more and more slowly with the increase of $\mu$ in the large $\mu$ region, which might signify the invalidness of the model for the description of large $\mu$ effects. The chiral phase diagram with magnetic field has been presented in Fig. 3, which shows clearly the influence of $\mu$ and $\mathcal{B}$ on $T_{\chi}$.

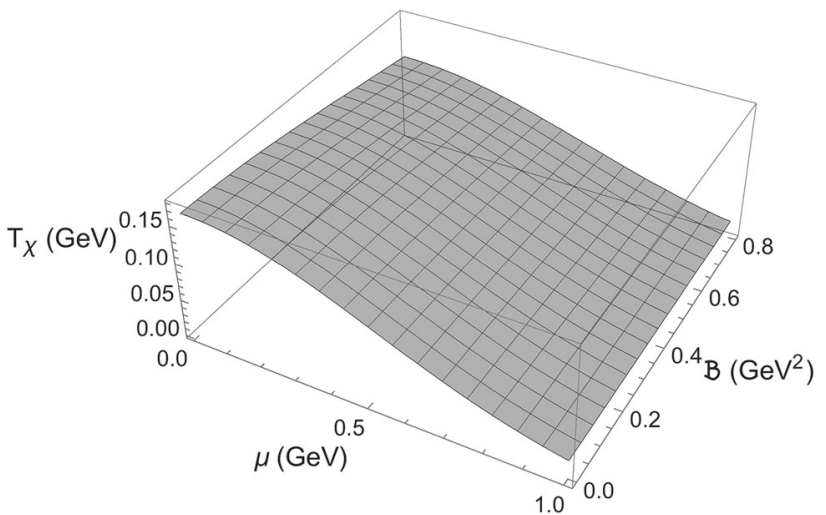

Fig. 3 The chiral phase diagram with magnetic field

\section{Melting properties of the vector and axial-vector mesons}

We now investigate the melting properties of the vector and axial-vector mesons by the thermal spectral functions in the charged magnetic background. In terms of the vector field 
$V=\frac{1}{2}\left(A_{L}+A_{R}\right)$ and the axial-vector field $A=\frac{1}{2}\left(A_{L}-\right.$ $A_{R}$ ), the gauge sector of the bulk action (1) can be rewritten as

$S_{v a}=-\frac{1}{2 g_{5}^{2}} \int d^{5} x \sqrt{-g} e^{-\Phi(z)} \operatorname{Tr}\left\{F_{V}^{2}+F_{A}^{2}\right\}$,

where the field strengths take the forms

$$
\begin{aligned}
& F_{V}^{M N}=\partial^{M} V^{N}-\partial^{N} V^{M}-\frac{i}{\sqrt{2}}\left[V^{M}, V^{N}\right], \\
& F_{A}^{M N}=\partial^{M} A^{N}-\partial^{N} A^{M}-\frac{i}{\sqrt{2}}\left[A^{M}, A^{N}\right] .
\end{aligned}
$$

Besides the action $S_{v a}$, the vector and axial-vector gauge field also appear in the covariant derivative of the bulk scalar field, which contributes to the EOM of the axial-vector meson.

\subsection{The spectral function for the vector meson}

We first consider the thermal spectral function for the vector meson. The EOM of the vector meson can be derived from the action (12) as

$\partial_{z}\left(e^{-\Phi} \sqrt{-g} g^{z z} g^{\mu \lambda} \partial_{z} V_{\lambda}\right)+e^{-\Phi} \sqrt{-g} g^{\mu \lambda} g^{\nu \rho} \partial_{\nu} \partial_{\rho} V_{\lambda}=0$

In terms of the metric ansatz (2), the spatial part of Eq. (14) in the momentum space can be written as

$$
\begin{aligned}
& V_{1,2}^{\prime \prime}+\left(A^{\prime}-\Phi^{\prime}-2 P^{\prime}+\frac{f^{\prime}}{f}\right) V_{1,2}^{\prime} \\
& +\frac{1}{f}\left(\frac{1}{f} \omega^{2}-e^{-2 P}\left(q_{1}^{2}+q_{2}^{2}\right)-e^{-2 Q} q_{3}^{2}\right) V_{1,2}=0 \\
& V_{3}^{\prime \prime}+\left(A^{\prime}-\Phi^{\prime}-2 Q^{\prime}+\frac{f^{\prime}}{f}\right) V_{3}^{\prime} \\
& +\frac{1}{f}\left(\frac{1}{f} \omega^{2}-e^{-2 P}\left(q_{1}^{2}+q_{2}^{2}\right)-e^{-2 Q_{2}^{2}}\right) V_{3}=0
\end{aligned}
$$

where the 4-momentum $p^{\mu}=\left(\omega, q_{1}, q_{2}, q_{3}\right)$ and $V_{i}^{\prime}=$ $\partial_{z} V_{i}(p, z)$ with $V_{i}(p, z)$ being the bulk-to-boundary propagator for the vector meson. Note that the EOM of the $x_{1,2}$ components $V_{1,2}(p, z)$ is different from that of the $x_{3}$ components $V_{3}(p, z)$ due to the existence of a magnetic field along the $x_{3}$ axis. To compute the spectral function for the vector meson, we will use Eq. (15) of the transverse components $V_{1,2}(p, z)$ with zero momentum $\left(q^{2}=0\right)$, which only differs from Eq. (16) qualitatively.

To further simplify the computation, we change the variables:

$z \rightarrow u z_{h}, \quad V_{1,2}(p, z) \rightarrow v(u), \quad 0<u<1$.
Then Eq. (15) with $q^{2}=0$ can be recast in the form:

$v^{\prime \prime}(u)+k_{1}(u) v^{\prime}(u)+k_{0}(u) v(u)=0$

with

$$
\begin{aligned}
k_{0}(u)= & \frac{\omega^{2} z_{h}^{2}}{\left(s(u)+\frac{2}{3} B^{2} z_{h}^{4} u^{4} \ln u\right)^{2}}, \\
k_{1}(u)= & \frac{1}{u}\left[3-2 \mu_{g}^{2} z_{h}^{2} u^{2}+\frac{4 B^{2} z_{h}^{4} u^{4} \ln u}{3 s(u)}\right. \\
& \left.-\frac{2\left(6-B^{2} z_{h}^{4} u^{4}-3 Q^{2} u^{6}\right)}{3 s(u)+2 B^{2} z_{h}^{4} u^{4} \ln u}\right],
\end{aligned}
$$

where $s(u)=\left(1-u^{2}\right)\left(1+u^{2}-Q^{2} u^{4}\right)$.

To solve Eq. (18), we need to fix the IR and UV boundary conditions. The IR asymptotic solution of Eq. (18) has a closed form:

$v(u \sim 1)=c_{+} \phi_{+}(u)+c_{-} \phi_{-}(u)$,

where the out-coming solution $\phi_{+}$and the in-falling one $\phi_{-}$ take the forms

$\phi_{ \pm}(u)=(1-u)^{ \pm \frac{3 \mathrm{i} \omega z_{h}}{2\left(6-3 Q^{2}-B^{2} z_{h}^{4}\right)}}$.

The UV asymptotic solution of Eq. (18) can be obtained as

$$
\begin{aligned}
v(u \sim 0)= & A\left(1+c_{l 2} u^{2} \log u+c_{l 4} u^{4} \log u+c_{4} u^{4}+\cdots\right) \\
& +B\left(u^{2}+d_{4} u^{4}+\cdots\right)
\end{aligned}
$$

where $A$ and $B$ are two arbitrary constants, and the coefficients $c_{l 2}, c_{l 4}, c_{4}$ and $d_{4}$ take the forms

$c_{l 2}=-\frac{1}{2} z_{h}^{2} \omega^{2}$

$c_{l 4}=-\frac{1}{16} z_{h}^{4} \omega^{2}\left(4 \mu_{g}^{2}-\omega^{2}\right)$,

$c_{4}=\frac{1}{64} z_{h}^{4} \omega^{2}\left(4 \mu_{g}^{2}-3 \omega^{2}\right)$,

$d_{4}=\frac{1}{8} z_{h}^{2}\left(4 \mu_{g}^{2}-\omega^{2}\right)$

Since the spectral function depends only on the ratio $B / A$, we just set $A=1$ in the numerical calculation. The constant $B$ can then be determined by solving Eq. (18) with the proper IR boundary condition.

To compute the spectral function, we need the retarded Green's function that corresponds to the IR in-falling solution $\phi_{-}$in holography $[96,97]$. According to [33], the retarded 
Green's function $D^{R}(\omega)$ is related to the UV asymptotics of $v(u)$ by

$$
\begin{aligned}
D^{R}(\omega) & =-\frac{C}{z_{h}^{2}} \lim _{u \rightarrow \epsilon}\left(\frac{1}{u} v^{*} v^{\prime}\right) \\
& =-\frac{2 C}{z_{h}^{2}}\left[B(\omega)+\frac{1}{2} c_{l 2}+c_{l 2} \log \epsilon\right],
\end{aligned}
$$

where $C=N_{c}^{2} /\left(64 \pi^{2} L\right)$ and $B(\omega)$ is just the constant in (22). The spectral function of the vector meson is determined by the imaginary part of $D^{R}(\omega)$ as

$\rho_{v}(\omega)=-\frac{1}{\pi} \operatorname{Im} D^{R}(\omega)=\frac{2 C}{\pi z_{h}^{2}} \operatorname{Im} B(\omega)$.

Given the IR in-falling boundary condition, we can solve Eq. (18) numerically and extract the value of $B(\omega)$ as a function of $\omega$, then the spectral function of the vector meson can be obtained.

4.2 The spectral function for the axial-vector meson

Now we consider the spectral function for the axial-vector meson, the EOM of which can be derived as

$$
\begin{aligned}
& \partial_{z}\left(e^{-\Phi} \sqrt{-g} g^{z z} g^{\mu \lambda} \partial_{z} A_{\lambda}\right)+e^{-\Phi} \sqrt{-g} g^{\mu \lambda} g^{\nu \rho} \partial_{\nu} \partial_{\rho} A_{\lambda} \\
& \quad-g_{5}^{2} e^{-\Phi} \sqrt{-g} \chi^{2} A^{\mu}=0 .
\end{aligned}
$$

The only difference from the EOM of the vector meson is the last term, which comes from the covariant derivative of the scalar field. Substituting the metric ansatz (2) into Eq. (26), we obtain the spatial part of the EOM in the momentum space,

$$
\begin{aligned}
& A_{1,2}^{\prime \prime}+\left(A^{\prime}-\Phi^{\prime}-2 P^{\prime}+\frac{f^{\prime}}{f}\right) A_{1,2}^{\prime} \\
& +\frac{1}{f}\left(\frac{1}{f} \omega^{2}-e^{-2 P}\left(q_{1}^{2}+q_{2}^{2}\right)-e^{-2 Q} q_{3}^{2}-g_{5}^{2} e^{2 A} \chi^{2}\right) A_{1,2}=0, \\
& A_{3}^{\prime \prime}+\left(A^{\prime}-\Phi^{\prime}-2 Q^{\prime}+\frac{f^{\prime}}{f}\right) A_{3}^{\prime} \\
& \quad+\frac{1}{f}\left(\frac{1}{f} \omega^{2}-e^{-2 P}\left(q_{1}^{2}+q_{2}^{2}\right)-e^{-2 Q} q_{3}^{2}-g_{5}^{2} e^{2 A} \chi^{2}\right) A_{3}=0,
\end{aligned}
$$

where $A_{i}^{\prime}=\partial_{z} A_{i}(p, z)$ with $A_{i}(p, z)$ being the bulk-toboundary propagator for the axial-vector meson. As in the vector case, we only use Eq. (27) of the transverse components with $q^{2}=0$ to compute the spectral function of the axial-vector meson.

With the replacement of variables:

$$
z \rightarrow u z_{h}, \quad A_{1,2}(p, z) \rightarrow a(u), \quad 0<u<1,
$$

Eq. (27) with $q^{2}=0$ can be recast in the form:

$a^{\prime \prime}(u)+l_{1}(u) a^{\prime}(u)+l_{0}(u) a(u)=0$,

where $l_{1}(u)=k_{1}(u)$ and

$l_{0}(u)=\frac{\omega^{2} z_{h}^{2} u^{2}-4 \pi^{2}\left(s(u)+\frac{2}{3} B^{2} z_{h}^{4} u^{4} \ln u\right) \chi^{2}}{u^{2}\left(s(u)+\frac{2}{3} B^{2} z_{h}^{4} u^{4} \ln u\right)^{2}}$.

The IR asymptotic solution of the axial-vector field $a(u)$ has the same form as that of the vector field:

$a(u \sim 1)=\tilde{c}_{+} \phi_{+}(u)+\tilde{c}_{-} \phi_{-}(u)$.

According to AdS/CFT, we choose the IR in-falling solution $\phi_{-}$as the physical one. The UV asymptotic form of $a(u)$ can be obtained as

$a(u \sim 0)=\tilde{A}\left(1+\tilde{c}_{l 2} u^{2} \log u+\cdots\right)+\tilde{B}\left(u^{2}+\cdots\right)$

with two arbitrary constants $\tilde{A}, \tilde{B}$ and $\tilde{c}_{l 2}=\frac{1}{2}$ $z_{h}^{2}\left(g_{5}^{2} m_{q}^{2} \zeta^{2}-\omega^{2}\right)$. We also set $\tilde{A}=1$ to fix the overall uncertainty, and solve Eq. (30) numerically under the given IR boundary condition.

The retarded Green's function in the axial-vector case is related to the UV asymptotics of $a(u)$ by

$$
\begin{aligned}
\tilde{D}^{R}(\omega) & =-\frac{C}{z_{h}^{2}} \lim _{u \rightarrow \epsilon}\left(\frac{1}{u} a^{*} a^{\prime}\right) \\
& =-\frac{2 C}{z_{h}^{2}}\left[\tilde{B}(\omega)+\frac{1}{2} \tilde{c}_{l 2}+\tilde{c}_{l 2} \log \epsilon\right],
\end{aligned}
$$

where $C=N_{c}^{2} /\left(64 \pi^{2} L\right)$ and $\tilde{B}(\omega)$ is the coefficient to be determined in (33). Similarly, the spectral function of the axial-vector meson can be written as

$\rho_{a}(\omega)=-\frac{1}{\pi} \operatorname{Im} \tilde{D}^{R}(\omega)=\frac{2 C}{\pi z_{h}^{2}} \operatorname{Im} \tilde{B}(\omega)$.

\subsection{Numerical results for the spectral functions}

With the proper boundary conditions, we solve Eqs. (18) and (30) numerically at given temperature $T$, chemical potential $\mu$ and magnetic field $\mathcal{B}$, from which the retarded Green's functions and the spectral functions for the vector and axialvector mesons can be obtained. The numerical results are presented in Figs. 4, 5 and 6. The spectral functions $\rho_{v}(\omega)$ and $\rho_{a}(\omega)$ for four different temperatures at $\mu=\mathcal{B}=0$ are plotted in Fig. 4, where we can see clearly the meson melting behaviors with the increase of temperature. The variations of $\rho_{v}(\omega)$ and $\rho_{a}(\omega)$ with $T$ have similar behaviors with a slight 
difference of the melting critical temperature. In Fig. 5, we show the spectral functions $\rho_{v}(\omega)$ and $\rho_{a}(\omega)$ for four different chemical potentials at fixed $\mathcal{B}$ and $T$, which resemble the variations of the spectral functions with different $T$, as compared with Fig. 4.

We display in Fig. 6 the spectral functions $\rho_{v}(\omega)$ and $\rho_{a}(\omega)$ for four different values of $\mathcal{B}$ at $\mu=0$ and $T=$ $10 \mathrm{MeV}$, which indicates that the magnetic field somehow can also cause the vector and axial-vector mesons to melt. However, one should be careful here, since the perturbative solution of the charged magnetic background cannot be pushed far to the region of very large $\mathcal{B}$. Here we only show the figures up to $\mathcal{B}=0.08 \mathrm{GeV}^{2}$ in the analysis of spectral functions.

To extract the melting critical temperature $T_{c}$, we may give a prescription for $T_{c}$ directly using the spectral functions, as in Ref. [61]. Here we adopt another method following Refs. [32,33]. With the field redefinition $V_{1,2}=$ $e^{(\Phi+2 P-A) / 2} f^{-1 / 2} \tilde{v}(z)$ and $A_{1,2}=e^{(\Phi+2 P-A) / 2} f^{-1 / 2} \tilde{a}(z)$, Eqs. (15) and (27) with $q^{2}=0$ can be recast in the form:

$\tilde{v}^{\prime \prime}-U_{v}(z) \tilde{v}=0, \quad \tilde{a}^{\prime \prime}-U_{a}(z) \tilde{a}=0$,

where the potential functions take the forms

$$
\begin{aligned}
U_{v}(z)= & \frac{1}{2}\left(A^{\prime \prime}-\Phi^{\prime \prime}-2 P^{\prime \prime}\right)+\frac{1}{4}\left(A^{\prime}-\Phi^{\prime}-2 P^{\prime}\right)^{2} \\
& +\frac{f^{\prime}}{2 f}\left(A^{\prime}-\Phi^{\prime}-2 P^{\prime}\right)-\frac{f^{\prime 2}}{4 f^{2}}+\frac{f^{\prime \prime}}{2 f}-\frac{\omega^{2}}{f^{2}}, \\
U_{a}(z)= & \frac{1}{2}\left(A^{\prime \prime}-\Phi^{\prime \prime}-2 P^{\prime \prime}\right)+\frac{1}{4}\left(A^{\prime}-\Phi^{\prime}-2 P^{\prime}\right)^{2} \\
& +\frac{f^{\prime}}{2 f}\left(A^{\prime}-\Phi^{\prime}-2 P^{\prime}\right)-\frac{f^{\prime 2}}{4 f^{2}}+\frac{f^{\prime \prime}}{2 f}-\frac{\omega^{2}}{f^{2}} \\
& +\frac{g_{5}^{2}}{f} e^{2 A} \chi^{2} .
\end{aligned}
$$

To guarantee the existence of bound states for the mesons, these potential functions should be convex around some region of $z$. The curves of potential functions for the vector and axial-vector mesons at four different temperatures are presented in Fig. 7, where the values of $\omega$ have been taken as the masses of the lowest-lying meson states [91]. We can see that $U_{v}(z)$ and $U_{a}(z)$ will become monotonic when the temperature increases to certain values, which can be defined as the melting critical temperature $T_{c}$. Besides the case of $\mu=\mathcal{B}=0$, we also show another two cases in Fig. 7, i.e., the case of $\mu=0, \mathcal{B}=0.03 \mathrm{GeV}^{2}$ and the case of $\mu=60 \mathrm{MeV}, \mathcal{B}=0$. These indicate that the influence of $\mu$ and $\mathcal{B}$ on the potential functions is similar to that of $T$.

By the method of potential function, we can extract the melting critical temperature $T_{c}$ at given chemical potential $\mu$ and magnetic field $\mathcal{B}$. In Fig. 8, we show the 3D phase diagrams for the melting of vector and axial-vector mesons. At $\mu=\mathcal{B}=0$, the melting critical temperature of vector meson is $T_{c} \simeq 59 \mathrm{MeV}$, while for axial-vector meson $T_{c} \simeq 97 \mathrm{MeV}$, which is larger than the melting temperature of vector meson in our model. Since we have adopted the method of potential function to extract the melting critical temperatures, the only difference between the vector and axial-vector case comes from the last term of $U_{a}(z)$, which contributes to the potential function of axial-vector meson. That is, the discrepancy of melting temperatures in our model results from a vacuum contribution to axial-vector meson. However, we cannot expect that the QCD phase transition should exhibit such a distinction for the melting temperatures of different mesons, although the vacuum part really plays a significant role in the mass spectra of axial-vector mesons. We may suspect that the vacuum effects which distinguish the melting temperatures of vector and axial-vector mesons may be suppressed by some unknown reasons.

We find that the melting temperatures of mesons are much smaller than the chiral transition temperature $T_{\chi} \simeq 165 \mathrm{MeV}$ in our model, as compared with Fig. 3. The dissociation of mesons is intimately related to the deconfinement, whose properties are mainly incorporated in the bulk background. However, the black hole solution used in our model is incapable of reproducing the equations of state which characterize the deconfining process. A more proper way is to use a bulk background which can mimic the QCD equations of state. For instance, such a background solution may be solved from an Einstein-Maxwell-Dilaton system [98-103]. As we know, QCD thermodynamics at lower temperatures around $100 \mathrm{MeV}$ or below is well described by a pressure of noninteracting free hadrons which is $1 / N_{c}$ suppressed with respect to the pressure of quark-gluon plasma at higher temperatures. Thus we should note that even an improved bulk background may not be enough to give a full description on both the deconfinement and chiral phase transition. At low temperatures, the string loop effects should be incorporated in order to characterize the thermodynamics of QCD.

\section{Summary and conclusion}

In this work, we studied the chiral transition and the melting properties of vector and axial-vector mesons at finite chemical potential under the influence of magnetic field in a simply improved soft-wall AdS/QCD model, which can realize consistently the spontaneous chiral symmetry breaking and the linear Regge spectra [91]. The background magnetic field is introduced by a charged magnetic black hole solution which is solved perturbatively from an Einstein-Maxwell system with a negative cosmological constant [92].

The effects of chemical potential $\mu$ and magnetic field $\mathcal{B}$ on chiral transition were investigated in detail. The transi- 

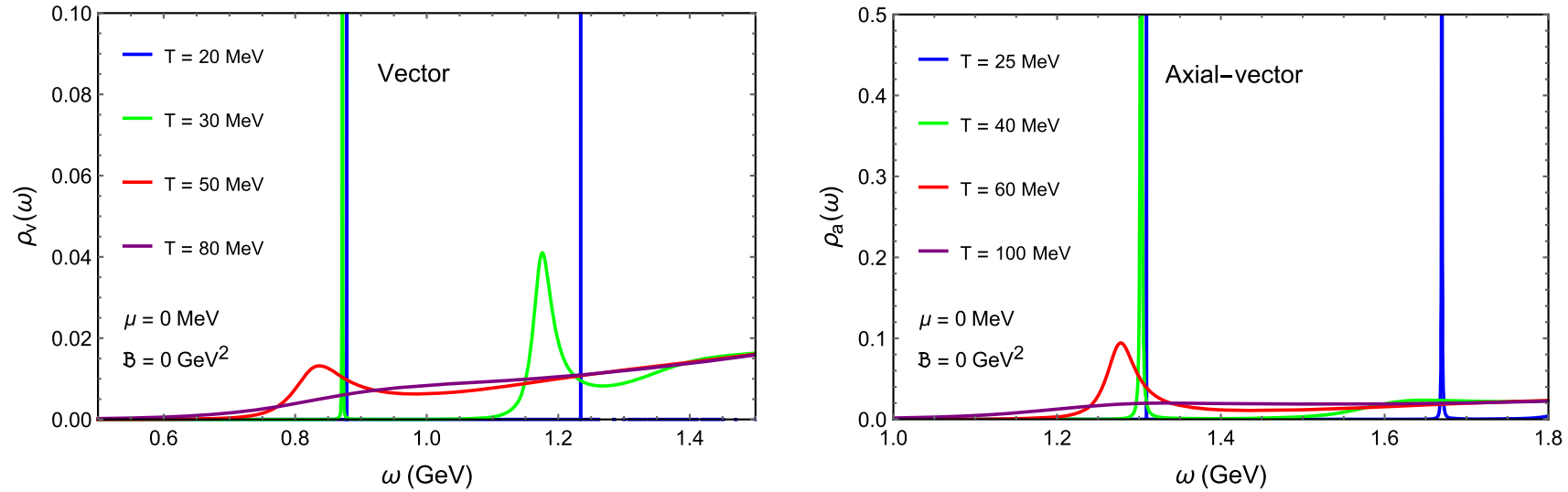

Fig. 4 The spectral functions $\rho_{v}(\omega)$ and $\rho_{a}(\omega)$ for four different temperatures at $\mu=\mathcal{B}=0$
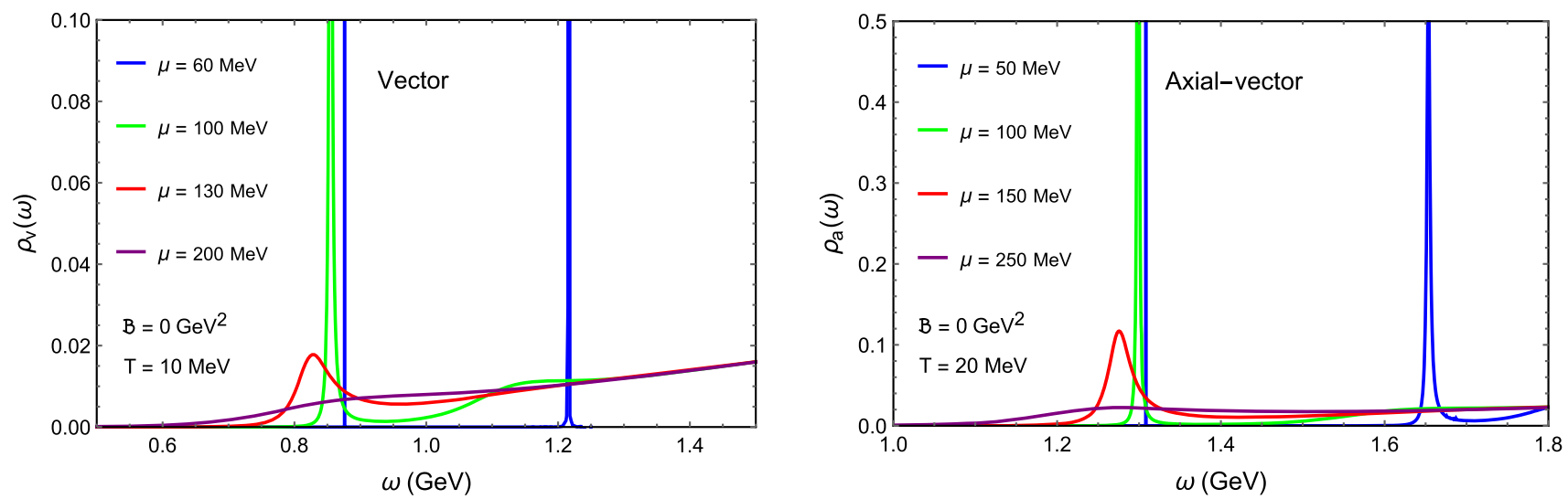

Fig. 5 The spectral functions $\rho_{v}(\omega)$ and $\rho_{a}(\omega)$ for four different chemical potentials at fixed $\mathcal{B}$ and $T$
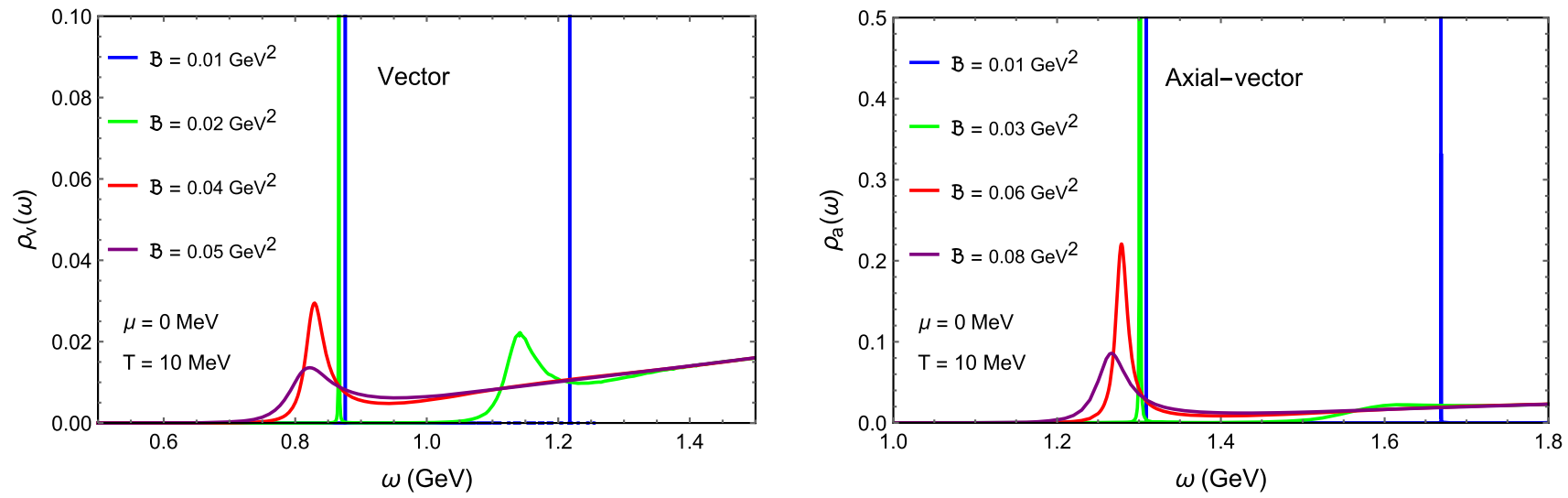

Fig. 6 The spectral functions $\rho_{v}(\omega)$ and $\rho_{a}(\omega)$ for four different values of $\mathcal{B}$ at $\mu=0$ and $T=10 \mathrm{MeV}$

tion temperatures $T_{\chi}$ have been extracted at each $\mu$ and $\mathcal{B}$, from which the chiral phase diagram has been obtained. Then we studied the effects of $\mu$ and $\mathcal{B}$ on the spectral functions of vector and axial-vector mesons. The melting critical temperatures $T_{c}$ have been obtained by the method of potential function, and the $3 \mathrm{D}$ phase diagrams have also been obtained from meson melting. We find that the inverse magnetic catal- ysis naturally emerged both in chiral transition and in the dissociation of vector and axial-vector mesons.

It has been shown that our model can give a reasonable chiral transition temperature $\left(T_{\chi} \simeq 165 \mathrm{MeV}\right)$ at $\mu=\mathcal{B}=0$. However, we find that $T_{\chi}$ decreases too slowly at large $\mu$, which seems not sensible. Furthermore, the chiral phase diagram obtained from our model contains no critical end point (CEP), which can be realized by introducing a coupling term 


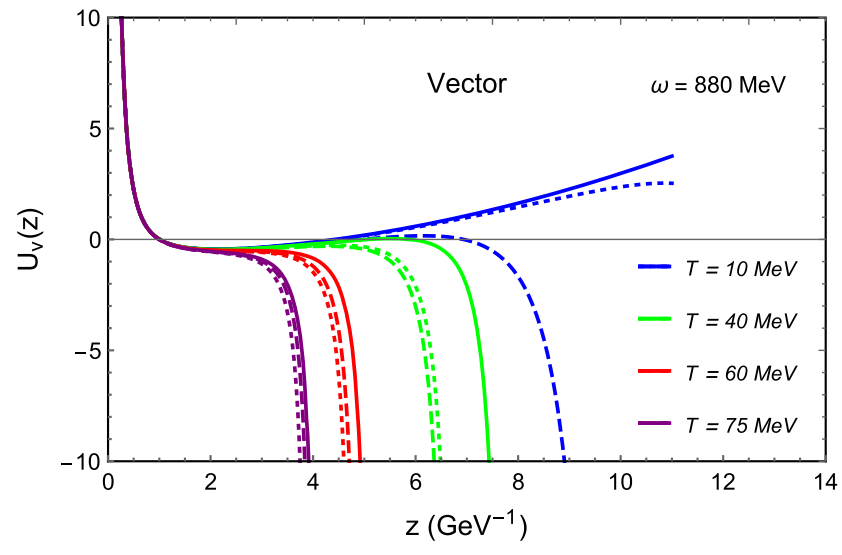

Fig. 7 The potential functions $U_{v}(z)$ and $U_{a}(z)$ for the ground states of the vector and axial-vector mesons at four different temperatures. The solid curves denote the case of $\mu=0$ and $\mathcal{B}=0$, the dashed curves

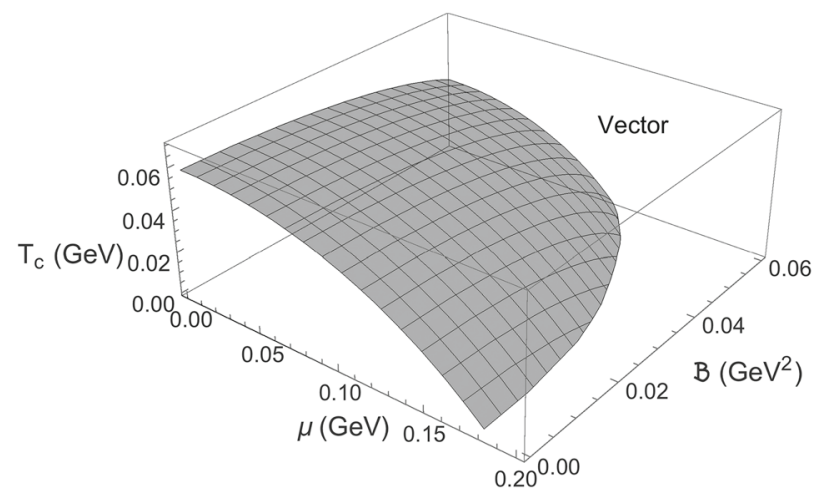

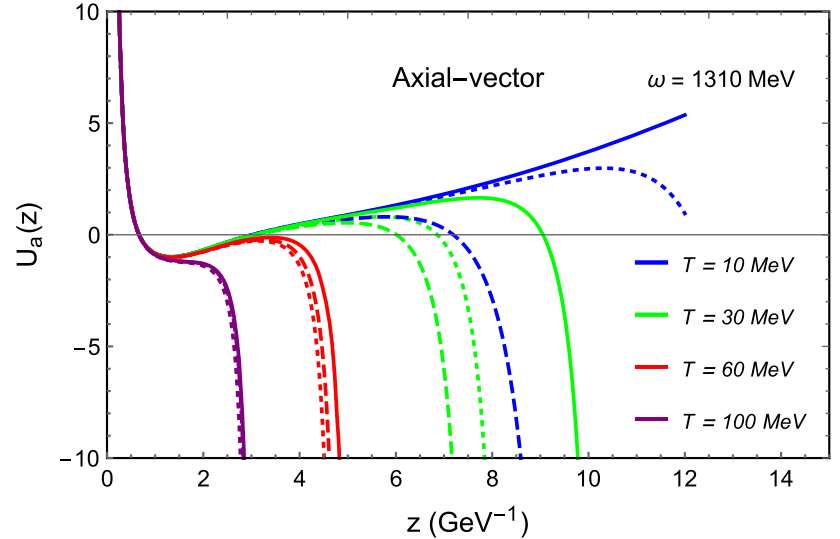

denote the case of $\mu=0$ and $\mathcal{B}=0.03 \mathrm{GeV}^{2}$, and the dotted curves denote the case of $\mu=60 \mathrm{MeV}$ and $\mathcal{B}=0$

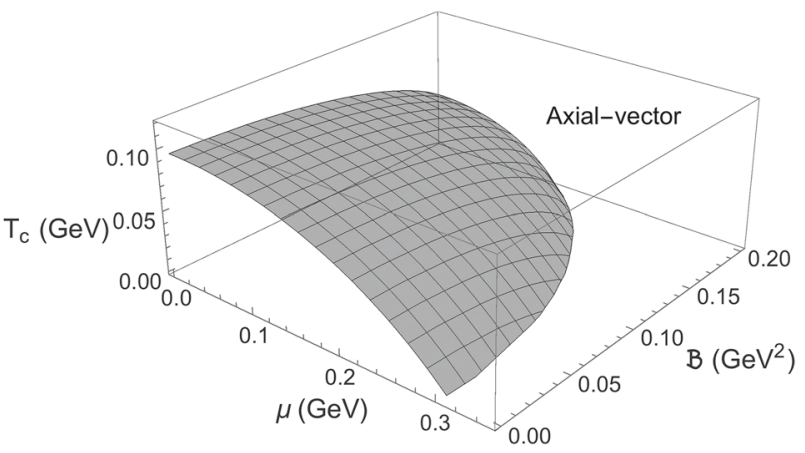

Fig. 8 The phase diagrams for the melting of vector meson (left panel) and axial-vector meson (right panel)

of the $U(1)$ gauge field and the bulk scalar field in the twoflavor case [104]. A natural generalization of this improved soft-wall model to the $2+1$ flavor case can also generate the CEP in the QCD phase diagram [77]. We also find that the melting critical temperatures $T_{c}$ for both vector and axialvector mesons are much smaller than the chiral transition temperature $T_{\chi}$ at $\mu=\mathcal{B}=0$. To address this issue, we should be aware that at low temperatures the string loop effects become important for the description of QCD thermodynamics. However, these effects have not been considered in most of holographic studies. In addition, since the black hole solution that we used cannot mimic the equations of state in QCD, we may resort to a more realistic bulk background in order to reconcile the chiral transition with the deconfinement that is closely related to meson dissociation.

Although we cannot trust the results of our model at large $\mathcal{B}$ since a perturbative background solution has been used, we do find that the spectral functions of vector and axialvector mesons become more and more flat with the increase of $\mathcal{B}$. Thus it seems that the magnetic fields can also generate melting behaviors of mesons. More discussions are neces- sary for us to get any solid conclusion about this point. As in Ref. [105], we should adopt an improved background solution to characterize QCD thermodynamics and especially the deconfinement phase transition. In order to incorporate the magnetic field in such an improved background, it may be easier to find the numerical solution instead of an analytical perturbative one. Further, we also need to consider the back-reaction of the flavor part to the bulk geometry in the following studies.

Acknowledgements This work is supported by the National Natural Science Foundation of China (NSFC) (Grant no. 11905055), the Natural Science Foundation of Hunan Province, China (Grant no. 2020JJ5026) and the Fundamental Research Funds for the Central Universities. In addition, this work is also partly supported by the NSFC (Grant nos. 11851302, 11851303, 11747601 and 11690022), and the Strategic Priority Research Program of the Chinese Academy of Sciences (Grant no. XDB23030100) as well as the CAS Center for Excellence in Particle Physics (CCEPP).

Data Availability Statement This manuscript has no associated data or the data will not be deposited. [Authors' comment: Indeed, the data of our work have all been displayed in the form of Figures in our paper, which are clear and vivid for the illustration of the results of our work.] 
Open Access This article is licensed under a Creative Commons Attribution 4.0 International License, which permits use, sharing, adaptation, distribution and reproduction in any medium or format, as long as you give appropriate credit to the original author(s) and the source, provide a link to the Creative Commons licence, and indicate if changes were made. The images or other third party material in this article are included in the article's Creative Commons licence, unless indicated otherwise in a credit line to the material. If material is not included in the article's Creative Commons licence and your intended use is not permitted by statutory regulation or exceeds the permitted use, you will need to obtain permission directly from the copyright holder. To view a copy of this licence, visit http://creativecomm ons.org/licenses/by/4.0/.

Funded by SCOAP 3 .

\section{Appendix}

Setup of a charged magnetic background

In our work, we have adopted a bulk gravitational background with electric charge density and uniform magnetic field on the boundary, which can be obtained by a 5D Einstein-Maxwell theory with a negative cosmological constant [92]. The action of this background system can be written as

$S_{E M}=\frac{1}{16 \pi G_{5}} \int d^{5} x \sqrt{-g}\left(R-F_{M N} F^{M N}+\frac{12}{L^{2}}\right)+S_{\text {bndy }}$,

where the boundary term $S_{\text {bndy }}$ is irrelevant in our consideration. The Einstein equation and the EOM of the $U(1)$ gauge field $A_{M}$ can be derived as

$R_{M N}=-\frac{1}{3} g_{M N} F_{I J} F^{I J}+2 F_{M}{ }^{K} F_{N K}-\frac{4}{L^{2}} g_{M N}$,

$\nabla_{M} F^{M N}=0$.

Following Ref. [92], we assume that the uniform magnetic field is along the $x_{3}$ direction and take the following ansatz:

$$
\begin{aligned}
d s^{2} & =-U(r) d t^{2}+\frac{d r^{2}}{U(r)}+e^{2 V(r)}\left(d x_{1}^{2}+d x_{2}^{2}\right)+e^{2 W(r)} d x_{3}^{2}, \\
F & =E(r) d r \wedge d t+B d x_{1} \wedge d x_{2},
\end{aligned}
$$

which preserves the symmetries of translation and rotation about the $x_{3}$ axis. The determinant of the metric is $g=-e^{4 V(r)+2 W(r)}$. In our calculation, we have used the perturbative form of Eq. (42) up to order $B^{2}$ with radial coordinate transformed to $z=1 / r$. This perturbative form of metric ansatz will be given in Eq. (66) below.

The nonzero component of Eq. (41) is

$$
0=\partial_{4}\left(\sqrt{-g} g^{4 M} g^{0 N} F_{M N}\right)=-\partial_{r}\left(e^{2 V(r)+W(r)} E(r)\right),
$$

which leads to solution $E(r)=\rho e^{-2 V(r)-W(r)}$ with the integral constant $\rho$ related to the charge of black hole (see below).
The nonzero components of Eq. (40) are

$$
\begin{aligned}
& U^{\prime \prime}+U^{\prime}\left(2 V^{\prime}+W^{\prime}\right)-\frac{8}{3} E^{2}-\frac{4}{3} B^{2} e^{-4 V}-\frac{8}{L^{2}}=0 \\
& U V^{\prime \prime}+U^{\prime} V^{\prime}+U V^{\prime}\left(2 V^{\prime}+W^{\prime}\right)+\frac{2}{3} E^{2} \\
& \quad+\frac{4}{3} B^{2} e^{-4 V}-\frac{4}{L^{2}}=0 \\
& U\left(V^{\prime \prime}-W^{\prime \prime}\right)+\left(U^{\prime}+U\left(2 V^{\prime}+W^{\prime}\right)\right)\left(V^{\prime}-W^{\prime}\right) \\
& \quad+2 B^{2} e^{-4 V}=0 \\
& U^{\prime}\left(2 V^{\prime}+W^{\prime}\right)+2 U V^{\prime 2}+4 U V^{\prime} W^{\prime}+2 E^{2} \\
& \quad+2 B^{2} e^{-4 V}-\frac{12}{L^{2}}=0,
\end{aligned}
$$

where the last one can be taken as a constraint equation. In our work, we only need the solution up to order $B^{2}$, so the Einstein equation will be solved perturbatively below.

For the case of $B=0$, we have $V(r)=W(r)$, then Eqs. (45)-(48) reduce to

$$
\begin{array}{r}
U^{\prime \prime}+3 U^{\prime} V^{\prime}-\frac{8}{3} E^{2}-\frac{8}{L^{2}}=0, \\
U V^{\prime \prime}+U^{\prime} V^{\prime}+3 U V^{\prime 2}+\frac{2}{3} E^{2}-\frac{4}{L^{2}}=0, \\
3 U^{\prime} V^{\prime}+6 U V^{\prime 2}+2 E^{2}-\frac{12}{L^{2}}=0 .
\end{array}
$$

The combination $3 \times(50)-(49)$ yields

$V^{\prime \prime}+V^{\prime 2}=0$

which has the solution $V(r)=\ln \left(r-C_{1}\right)+C_{2}$. Physically, we shall take $C_{1}=C_{2}=0$, which leads to the AdS/ReissnerNördstrom (AdS/RN) black-hole solution:

$V_{0}(r)=\ln r, \quad E_{0}(r)=\rho r^{-3}$,

$U_{0}(r)=\frac{r^{2}}{L^{2}}+\frac{\rho^{2}}{3 r^{4}}-\frac{M}{r^{2}}=r^{2}+\frac{q^{2}}{r^{4}}-\frac{M}{r^{2}}$,

where $q=\frac{\rho}{\sqrt{3}}$ is the charge of the black hole, and the subscript 0 denotes the case of $B=0$.

Background solution up to order $B^{2}$

Now we consider the perturbative solution up to order $B^{2}$. The charged magnetic AdS black hole solution can be written as

$E=E_{0}+B^{2} E_{2}, \quad U=U_{0}+B^{2} U_{2}$,

$V=V_{0}+B^{2} V_{2}, \quad W=V_{0}+B^{2} W_{2}$.

To separate out the scalar and tensor perturbative corrections, we introduce the combinations $S_{2}=2 V_{2}+W_{2}$ and 
$T_{2}=V_{2}-W_{2}$ [92]. Then Eq. (47) to order $B^{2}$ (with $V_{0}=\ln r$ substituted) reduces to

$\left(r^{3} U_{0} T_{2}^{\prime}\right)^{\prime}+\frac{2}{r}=0$

With the constraints $T_{2}(r \rightarrow \infty)=0$ and $T_{2}^{\prime}$ being smooth at the horizon $r_{h}$, we can solve Eq. (55) by two successive integrations,

$$
\begin{aligned}
T_{2}(r) & =\int_{\infty}^{r} d r^{\prime} \frac{1}{r^{\prime 3} U_{0}\left(r^{\prime}\right)} \int_{r_{h}}^{r^{\prime}} d r^{\prime \prime}\left(-\frac{2}{r^{\prime \prime}}\right) \\
& =\int_{\infty}^{r} d r^{\prime} \frac{-2 \ln \left(r^{\prime} / r_{h}\right)}{r^{\prime 3} U_{0}\left(r^{\prime}\right)} .
\end{aligned}
$$

The linear combination $3 \times(46)-(48)$ to order $B^{2}$ yields an equation of $S_{2}$,

$U_{0} S_{2}^{\prime \prime}+U_{0}^{\prime} T_{2}^{\prime}+U_{0} T_{2}^{\prime \prime}+\frac{2 U_{0} S_{2}^{\prime}}{r}+\frac{3 U_{0} T_{2}^{\prime}}{r}+\frac{2}{r^{4}}=0$,

which can be recast in the form

$$
\left(r^{2} S_{2}^{\prime}\right)^{\prime}=-\left(\frac{2}{r^{2} U_{0}}+\frac{r^{2} T_{2}^{\prime} U_{0}^{\prime}}{U_{0}}+3 r T_{2}^{\prime}+r^{2} T_{2}^{\prime \prime}\right) .
$$

It is easy to see that the right side of Eq. (58) vanishes when substituting (56) into it. Thus we have $\left(r^{2} S_{2}^{\prime}\right)^{\prime}=0$, which leads to $S_{2}(r)=C_{3} / r$. Then the function $E(r)$ to order $B^{2}$ can be obtained as

$$
\begin{gathered}
E(r)=\rho e^{-2 V(r)-W(r)}=\rho e^{-3 V_{0}(r)-B^{2} S_{2}(r)} \\
\simeq E_{0}(r)+B^{2} E_{2}(r)
\end{gathered}
$$

with

$$
E_{2}(r)=-\rho e^{-3 V_{0}(r)} S_{2}(r)=-C_{3} \rho r^{-4} .
$$

As in Ref. [92], we take $C_{3}=0$, then $S_{2}=E_{2}=0$. Thus we have $E(r)=E_{0}(r)$ and

$$
\begin{aligned}
V_{2} & =\frac{1}{3} T_{2}=\frac{-2}{3} \int_{\infty}^{r} d r^{\prime} \frac{\ln \left(r^{\prime} / r_{h}\right)}{r^{\prime 3} U_{0}\left(r^{\prime}\right)} \\
W_{2} & =-\frac{2}{3} T_{2}=\frac{4}{3} \int_{\infty}^{r} d r^{\prime} \frac{\ln \left(r^{\prime} / r_{h}\right)}{r^{\prime 3} U_{0}\left(r^{\prime}\right)} .
\end{aligned}
$$

Note that $W_{2}(r)=-2 V_{2}(r)$. As $E(r)=F_{40}=\partial_{r} A_{0}(r)$, the time component of the $U(1)$ gauge field can be obtained as

$$
A_{0}(r)=\int_{\infty}^{r} \frac{\rho}{r^{3}} d r+\mu=\mu-\frac{\rho}{2 r^{2}}=\mu-\frac{\sqrt{3}}{2} \frac{q}{r^{2}}
$$

where $\mu$ denotes the chemical potential of the system. We demands that $A_{0}\left(r_{h}\right)=0$ at the horizon $r_{h}$, hence $\mu$ is related to the charge of black hole by $\mu=\frac{\sqrt{3}}{2} \frac{q}{r_{h}^{2}}=\frac{\sqrt{3}}{2} q z_{h}^{2}$.

Equation (45) to order $B^{2}$ determines the function $U_{2}(r)$,

$U_{2}^{\prime \prime}+3 V_{0}^{\prime} U_{2}^{\prime}+U_{0}^{\prime} S_{2}^{\prime}-\frac{16}{3} E_{0} E_{2}-\frac{4}{3} e^{-4 V_{0}}=0$.

In terms of $S_{2}=E_{2}=0$ and $V_{0}=\ln r$, Eq. (63) can be written as

$U_{2}^{\prime \prime}+\frac{3}{r} U_{2}^{\prime}-\frac{4}{3 r^{4}}=0$

which, under the condition $U_{2}(r \rightarrow \infty)=0$, has the solution:

$U_{2}(r)=-\frac{2 \ln r}{3 r^{2}}-\frac{1}{3 r^{2}}-\frac{a_{3}}{2 r^{2}}$.

With the coordinate $z=1 / r$, the metric ansatz (42) for the charged magnetic background up to order $B^{2}$ takes the form

$$
\begin{aligned}
d s^{2}= & -U(r) d t^{2}+\frac{d r^{2}}{U(r)}+e^{2 V(r)}\left(d x_{1}^{2}+d x_{2}^{2}\right) \\
& +e^{2 W(r)} d x_{3}^{2} \\
= & e^{2 A(z)}\left(-f(z) d t^{2}+\frac{d z^{2}}{f(z)}+e^{2 P(z)}\left(d x_{1}^{2}\right.\right. \\
& \left.\left.+d x_{2}^{2}\right)+e^{2 Q(z)} d x_{3}^{2}\right),
\end{aligned}
$$

where

$$
\begin{aligned}
& A(z)=-\ln z, \quad f(z)=z^{2} U(1 / z), \\
& P(z)=B^{2} V_{2}(1 / z), \quad Q(z)=B^{2} W_{2}(1 / z),
\end{aligned}
$$

which reduces to the AdS/RN black hole for $B=0$. The determinant of this metric is

$g=-e^{10 A(z)} e^{4 B^{2} V_{2}(1 / z)+2 B^{2} W_{2}(1 / z)}=-e^{10 A(z)}$.

The Hawking temperature

We write the function $f(z)$ explicitly

$f(z)=z^{2} U(1 / z)=1-\tilde{M} z^{4}+q^{2} z^{6}+\frac{2 B^{2}}{3} z^{4} \ln z$

where $\tilde{M}=M+\frac{B^{2}}{3}+\frac{a_{3} B^{2}}{2}$. The condition that $f\left(z_{h}\right)=0$ at the horizon $z_{h}$ yields

$\tilde{M}=q^{2} z_{h}^{2}+\frac{1}{z_{h}^{4}}+\frac{2}{3} B^{2} \ln z_{h}$. 
Hence $f(z)$ can be rewritten as

$$
f(z)=1-\left(1+Q^{2}\right)\left(\frac{z}{z_{h}}\right)^{4}+Q^{2}\left(\frac{z}{z_{h}}\right)^{6}+\frac{2}{3} B^{2} z^{4} \ln \frac{z}{z_{h}}
$$

with $Q=q z_{h}^{3}$. The Hawking temperature is determined by

$$
T=\left|\frac{f^{\prime}\left(z_{h}\right)}{4 \pi}\right|=\frac{1}{\pi z_{h}}\left|1-\frac{2 \mu^{2} z_{h}^{2}}{3}-\frac{B^{2} z_{h}^{4}}{6}\right| .
$$

\section{References}

1. D. Kharzeev, K. Landsteiner, A. Schmitt, H.-U. Yee, (eds.), Strongly Interacting Matter in Magnetic Fields, vol. 871 (2013). https://doi.org/10.1007/978-3-642-37305-3

2. T. Vachaspati, Phys. Lett. B 265, 258 (1991). https://doi.org/10. 1016/0370-2693(91)90051-Q

3. D.E. Kharzeev, L.D. McLerran, H.J. Warringa, Nucl. Phys. A 803, 227 (2008). https://doi.org/10.1016/j.nuclphysa.2008.02. 298. arXiv:0711.0950 [hep-ph]

4. V. Gusynin, V. Miransky, I. Shovkovy, Phys. Rev. Lett. 73, 3499 (1994). https://doi.org/10.1103/PhysRevLett.73.3499. arXiv:hep-ph/9405262 (Erratum: Phys. Rev. Lett. 76, 1005 (1996))

5. A. Osipov, B. Hiller, A. Blin, J. da Providencia, Phys. Lett. B 650, 262 (2007). https://doi.org/10.1016/j.physletb.2007.05.033. arXiv:hep-ph/0701090

6. R. Gatto, M. Ruggieri, Phys. Rev. D 83, 034016 (2011). https:// doi.org/10.1103/PhysRevD.83.034016. arXiv:1012.1291 [hep$\mathrm{ph}]$

7. N. Callebaut, D. Dudal, H. Verschelde, Acta Phys. Pol. Suppl. 4, 671 (2011). https://doi.org/10.5506/APhysPolBSupp.4.671

8. G.S. Bali, F. Bruckmann, G. Endrodi, Z. Fodor, S.D. Katz, S. Krieg, A. Schafer, K.K. Szabo, JHEP 02, 044 (2012). https://doi. org/10.1007/JHEP02(2012)044. arXiv:1111.4956 [hep-lat]

9. G.S. Bali, F. Bruckmann, G. Endrodi, Z. Fodor, S.D. Katz, A. Schafer, Phys. Rev. D 86, 071502 (2012b). https://doi.org/10. 1103/PhysRevD.86.071502. arXiv:1206.4205 [hep-lat]

10. N. Agasian, S. Fedorov, Phys. Lett. B 663, 445 (2008). https://doi. org/10.1016/j.physletb.2008.04.050. arXiv:0803.3156 [hep-ph]

11. E.S. Fraga, L.F. Palhares, Phys. Rev. D 86, 016008 (2012). https://doi.org/10.1103/PhysRevD.86.016008. arXiv:1201.5881 [hep-ph]

12. M. Ferreira, P. Costa, O. Lourenço, T. Frederico, C. Providência, Phys. Rev. D 89, 116011 (2014). https://doi.org/10.1103/ PhysRevD.89.116011. arXiv:1404.5577 [hep-ph]

13. E.S. Fraga, A.J. Mizher, Phys. Rev. D 78, 025016 (2008). https://doi.org/10.1103/PhysRevD.78.025016. arXiv:0804.1452 [hep-ph]

14. K. Fukushima, Y. Hidaka, Phys. Rev. Lett. 110, 031601 (2013). https://doi.org/10.1103/PhysRevLett.110.031601. arXiv:1209.1319 [hep-ph]

15. J. Chao, P. Chu, M. Huang, Phys. Rev. D 88, 054009 (2013). https://doi.org/10.1103/PhysRevD.88.054009. arXiv:1305.1100 [hep-ph]

16. J.M. Maldacena, Int. J. Theor. Phys. 38, 1113 (1999). https://doi. org/10.1023/A:1026654312961. arXiv:hep-th/9711200

17. J.M. Maldacena, Adv. Theor. Math. Phys. 2, 231 (1998). https:// doi.org/10.4310/ATMP.1998.v2.n2.a1

18. S.S. Gubser, I.R. Klebanov, A.M. Polyakov, Phys. Lett. B 428, 105 (1998). https://doi.org/10.1016/S0370-2693(98)00377-3. arXiv:hep-th/9802109
19. E. Witten, Adv. Theor. Math. Phys. 2, 253 (1998). https://doi.org/ 10.4310/ATMP.1998.v2.n2.a2. arXiv:hep-th/9802150

20. M. Kruczenski, D. Mateos, R.C. Myers, D.J. Winters, JHEP 05, 041 (2004). https://doi.org/10.1088/1126-6708/2004/05/041. arXiv:hep-th/0311270

21. T. Sakai, S. Sugimoto, Prog. Theor. Phys. 113, 843 (2005a). https://doi.org/10.1143/PTP.113.843. arXiv:hep-th/0412141

22. T. Sakai, S. Sugimoto, Prog. Theor. Phys. 114, 1083 (2005b). https://doi.org/10.1143/PTP.114.1083. arXiv:hep-th/050707

23. L. Da Rold, A. Pomarol, Nucl. Phys. B 721, 79 (2005). https://doi. org/10.1016/j.nuclphysb.2005.05.009. arXiv:hep-ph/0501218

24. J. Erlich, E. Katz, D.T. Son, M.A. Stephanov, Phys. Rev. Lett. 95, 261602 (2005). https://doi.org/10.1103/PhysRevLett.95.261602. arXiv:hep-ph/0501128

25. G.F. de Teramond, S.J. Brodsky, Phys. Rev. Lett. 94, 201601 (2005). https://doi.org/10.1103/PhysRevLett.94.201601. arXiv:hep-th/0501022

26. A. Karch, E. Katz, D.T. Son, M.A. Stephanov, Phys. Rev. D 74, 015005 (2006). https://doi.org/10.1103/PhysRevD.74. 015005. arXiv:hep-ph/0602229

27. C. Csaki, M. Reece, JHEP 05, 062 (2007). https://doi.org/10. 1088/1126-6708/2007/05/062. arXiv:hep-ph/0608266

28. A. Cherman, T.D. Cohen, E.S. Werbos, Phys. Rev. C 79, 045203 (2009). https://doi.org/10.1103/PhysRevC.79.045203. arXiv:0804.1096 [hep-ph]

29. T. Gherghetta, J.I. Kapusta, T.M. Kelley, Phys. Rev. D 79, 076003 (2009). https://doi.org/10.1103/PhysRevD.79.076003. arXiv:0902.1998 [hep-ph]

30. Y.-Q. Sui, Y.-L. Wu, Z.-F. Xie, Y.-B. Yang, Phys. Rev. D 81, 014024 (2010). https://doi.org/10.1103/PhysRevD.81.014024. arXiv:0909.3887 [hep-ph]

31. P. Colangelo, F. Giannuzzi, S. Nicotri, Phys. Rev. D 80, 094019 (2009). https://doi.org/10.1103/PhysRevD.80.094019. arXiv:0909.1534 [hep-ph]

32. M. Fujita, K. Fukushima, T. Misumi, M. Murata, Phys. Rev. D 80, 035001 (2009). https://doi.org/10.1103/PhysRevD.80. 035001. arXiv:0903.2316 [hep-ph]

33. M. Fujita, T. Kikuchi, K. Fukushima, T. Misumi, M. Murata, Phys. Rev. D 81, 065024 (2010). https://doi.org/10.1103/PhysRevD.81. 065024. arXiv:0911.2298 [hep-ph]

34. T.M. Kelley, S.P. Bartz, J.I. Kapusta, Phys. Rev. D 83, 016002 (2011). https://doi.org/10.1103/PhysRevD.83.016002. arXiv:1009.3009 [hep-ph]

35. Y.-Q. Sui, Y.-L. Wu, Y.-B. Yang, Phys. Rev. D 83, 065030 (2011). https://doi.org/10.1103/PhysRevD.83.065030. arXiv:1012.3518 [hep-ph]

36. P. Colangelo, F. Giannuzzi, S. Nicotri, V. Tangorra, Eur. Phys. J. C 72, 2096 (2012). https://doi.org/10.1140/epjc/ s10052-012-2096-9. arXiv:1112.4402 [hep-ph]

37. D. Li, M. Huang, Q.-S. Yan, Eur. Phys. J. C 73, 2615 (2013). https://doi.org/10.1140/epjc/s10052-013-2615-3. arXiv:1206.2824 [hep-th]

38. L.-X. Cui, Z. Fang, Y.-L. Wu, Eur. Phys. J. C 76, 22 (2016a). https://doi.org/10.1140/epjc/s10052-015-3866-y. arXiv: 1310.6487 [hep-ph]

39. D. Li, M. Huang, JHEP 11, 088 (2013). https://doi.org/10.1007/ JHEP11(2013)088. arXiv:1303.6929 [hep-ph]

40. S.J. Brodsky, G.F. de Teramond, H.G. Dosch, J. Erlich, Phys. Rept. 584, 1 (2015). https://doi.org/10.1016/j.physrep.2015.05. 001. arXiv:1407.8131 [hep-ph]

41. Z. Fang, D. Li, Y.-L. Wu, Phys. Lett. B 754, 343 (2016). https:// doi.org/10.1016/j.physletb.2016.01.045. arXiv:1602.00379 [hep$\mathrm{ph}]$

42. Z. Fang, Phys. Rev. D 94, 074017 (2016a). https://doi.org/10. 1103/PhysRevD.94.074017. arXiv:1607.06197 [hep-ph] 
43. E.V. Shuryak, Quark gluon plasma. New discoveries at RHIC: a case of strongly interacting quark gluon plasma. Proceedings, RBRC workshop, Brookhaven, Upton, USA, May 14-15, 2004. Nucl. Phys. A 750, 64 (2005). https://doi.org/10.1016/j. nuclphysa.2004.10.022. arXiv:hep-ph/0405066

44. M.J. Tannenbaum, Rep. Prog. Phys. 69, 2005 (2006). https://doi. org/10.1088/0034-4885/69/7/R01. arXiv:nucl-ex/0603003

45. G. Policastro, D.T. Son, A.O. Starinets, Phys. Rev. Lett. 87, 081601 (2001). https://doi.org/10.1103/PhysRevLett.87.081601. arXiv:hep-th/0104066

46. R.-G. Cai, Z.-Y. Nie, N. Ohta, Y.-W. Sun, Phys. Rev. D 79, 066004 (2009). https://doi.org/10.1103/PhysRevD.79.066004. arXiv:0901.1421 [hep-th]

47. R.-G. Cai, Z.-Y. Nie, Y.-W. Sun, Phys. Rev. D 78, 126007 (2008). https://doi.org/10.1103/PhysRevD.78.126007. arXiv:0811.1665 [hep-th]

48. S.-J. Sin, I. Zahed, Phys. Lett. B 608, 265 (2005). https://doi.org/ 10.1016/j.physletb.2005.01.020. arXiv:hep-th/0407215

49. E. Shuryak, S.-J. Sin, I. Zahed, J. Korean Phys. Soc. 50, 384 (2007). https://doi.org/10.3938/jkps.50.384. arXiv:hep-th/0511199

50. H. Nastase, (2005). arXiv:hep-th/0501068 [hep-th]

51. R.A. Janik, R.B. Peschanski, Phys. Rev. D 73, 045013 (2006). https://doi.org/10.1103/PhysRevD.73.045013. arXiv:hep-th/0512162

52. C.P. Herzog, A. Karch, P. Kovtun, C. Kozcaz, L.G. Yaffe, JHEP 07, 013 (2006). https://doi.org/10.1088/1126-6708/2006/07/013. arXiv:hep-th/0605158

53. S. Nakamura, S.-J. Sin, JHEP 09, 020 (2006). https://doi.org/10. 1088/1126-6708/2006/09/020. arXiv:hep-th/0607123

54. S.-J. Sin, S. Nakamura, S.P. Kim, JHEP 12, 075 (2006). https:// doi.org/10.1088/1126-6708/2006/12/075. arXiv:hep-th/0610113

55. U. Gursoy, E. Kiritsis, JHEP 02, 032 (2008). https://doi.org/10. 1088/1126-6708/2008/02/032. arXiv:0707.1324 [hep-th]

56. U. Gursoy, E. Kiritsis, F. Nitti, JHEP 02, 019 (2008). https://doi. org/10.1088/1126-6708/2008/02/019. arXiv:0707.1349 [hep-th]

57. U. Gursoy, E. Kiritsis, L. Mazzanti, F. Nitti, Phys. Rev. Lett. 101, 181601 (2008b). https://doi.org/10.1103/PhysRevLett.101. 181601. arXiv:0804.0899 [hep-th]

58. U. Gursoy, E. Kiritsis, L. Mazzanti, F. Nitti, JHEP 05, 033 (2009). https://doi.org/10.1088/1126-6708/2009/05/033. arXiv:0812.0792 [hep-th]

59. D. Li, J. Liao, M. Huang, Phys. Rev. D 89, 126006 (2014). https://doi.org/10.1103/PhysRevD.89.126006. arXiv:1401.2035 [hep-ph]

60. D. Li, S. He, M. Huang, JHEP 06, 046 (2015). https://doi.org/10. 1007/JHEP06(2015)046. arXiv:1411.5332 [hep-ph]

61. L.-X. Cui, Z. Fang, Y.-L. Wu, Chin. Phys. C 40, 063101 (2016b). https://doi.org/10.1088/1674-1137/40/6/063101. arXiv:1404.0761 [hep-ph]

62. K. Chelabi, Z. Fang, M. Huang, D. Li, Y.-L. Wu, JHEP 04, 036 (2016). https://doi.org/10.1007/JHEP04(2016)036. arXiv:1512.06493 [hep-ph]

63. K. Chelabi, Z. Fang, M. Huang, D. Li, Y.-L. Wu, Phys. Rev. D 93, 101901 (2016b). https://doi.org/10.1103/PhysRevD.93.101901. arXiv:1511.02721 [hep-ph]

64. Z. Fang, S. He, D. Li, Nucl. Phys. B 907, 187 (2016). https://doi. org/10.1016/j.nuclphysb.2016.04.003. arXiv:1512.04062 [hep$\mathrm{ph}]$

65. D. Li, M. Huang, JHEP 02, 042 (2017). https://doi.org/10.1007/ JHEP02(2017)042. arXiv:1610.09814 [hep-ph]

66. Z. Fang, Phys. Lett. B 758, 1 (2016). https://doi.org/10.1016/j. physletb.2016.04.044

67. K.A. Mamo, Phys. Rev. D 94, 041901 (2016). https://doi.org/10. 1103/PhysRevD.94.041901. arXiv:1606.01598 [hep-th]
68. D. Dudal, S. Mahapatra, JHEP 04, 031 (2017). https://doi.org/10. 1007/JHEP04(2017)031. arXiv:1612.06248 [hep-th]

69. S.P. Bartz, T. Jacobson, Phys. Rev. D 94, 075022 (2016). https:// doi.org/10.1103/PhysRevD.94.075022. arXiv:1607.05751 [hep$\mathrm{ph}]$

70. S.P. Bartz, T. Jacobson, Phys. Rev. C 97, 044908 (2018). https:// doi.org/10.1103/PhysRevC.97.044908. arXiv:1801.00358 [hep$\mathrm{ph}]$

71. A. Ballon-Bayona, M. Ihl, J.P. Shock, D. Zoakos, JHEP 10, 038 (2017). https://doi.org/10.1007/JHEP10(2017)038. arXiv:1706.05977 [hep-th]

72. M. Attems, J. Casalderrey-Solana, D. Mateos, I. Papadimitriou, D. Santos-Oliván, C.F. Sopuerta, M. Triana, M. Zilhão, JHEP 10, 155 (2016). https://doi.org/10.1007/JHEP10(2016)155. arXiv:1603.01254 [hep-th]

73. M. Attems, J. Casalderrey-Solana, D. Mateos, D. Santos-Oliván, C.F. Sopuerta, M. Triana, M.Zilhão, JHEP 06, 154 (2017). https:// doi.org/10.1007/JHEP06(2017)154. arXiv:1703.09681 [hep-th]

74. D. Dudal, T.G. Mertens, Phys. Rev. D 97, 054035 (2018). https:// doi.org/10.1103/PhysRevD.97.054035. arXiv:1802.02805 [hepth]

75. J. Chen, S. He, M. Huang, D. Li, JHEP 01, 165 (2019). https:// doi.org/10.1007/JHEP01(2019)165. arXiv:1810.07019 [hep-ph]

76. Z. Fang, Y.-L. Wu, L. Zhang, Phys. Rev. D 98, 114003 (2018). $\quad$ https://doi.org/10.1103/PhysRevD.98.114003. arXiv:1805.05019 [hep-ph]

77. Z. Fang, Y.-L. Wu, L. Zhang, Phys. Rev. D 99, 034028 (2019a). $\quad$ https://doi.org/10.1103/PhysRevD.99.034028. arXiv:1810.12525 [hep-ph]

78. Z. Fang, Y.-L. Wu, L. Zhang, Phys. Rev. D 100, 054008 (2019b). https://doi.org/10.1103/PhysRevD.100.054008. arXiv:1904.04695 [hep-ph]

79. O. Bergman, G. Lifschytz, M. Lippert, JHEP 05, 007 (2008). https://doi.org/10.1088/1126-6708/2008/05/007. arXiv:0802.3720 [hep-th]

80. C.V. Johnson, A. Kundu, JHEP 12, 053 (2008). https://doi.org/ 10.1088/1126-6708/2008/12/053. arXiv:0803.0038 [hep-th]

81. V.G. Filev, R.C. Raskov, Adv. High Energy Phys. 2010, 473206 (2010). https://doi.org/10.1155/2010/473206. arXiv:1010.0444 [hep-th]

82. S. Bolognesi, D. Tong, Class. Quant. Grav. 29, 194003 (2012). https://doi.org/10.1088/0264-9381/29/19/194003. arXiv: 1110.5902 [hep-th]

83. A. Ballon-Bayona, JHEP 11, 168 (2013). https://doi.org/10.1007/ JHEP11(2013)168. arXiv:1307.6498 [hep-th]

84. K.A. Mamo, JHEP 05, 121 (2015). https://doi.org/10.1007/ JHEP05(2015)121. arXiv:1501.03262 [hep-th]

85. R. Rougemont, R. Critelli, J. Noronha, Phys. Rev. D 93, 045013 (2016a). https://doi.org/10.1103/PhysRevD.93.045013. arXiv:1505.07894 [hep-th]

86. D. Dudal, D.R. Granado, T.G. Mertens, Phys. Rev. D 93, 125004 (2016). https://doi.org/10.1103/PhysRevD.93.125004. arXiv: 1511.04042 [hep-th]

87. D.M. Rodrigues, E. Folco Capossoli, H. Boschi-Filho, Phys. Lett. B 780, 37 (2018). https://doi.org/10.1016/j.physletb.2018.02.049. arXiv:1709.09258 [hep-th]

88. F. Preis, A. Rebhan, A. Schmitt, JHEP 03, 033 (2011). https://doi. org/10.1007/JHEP03(2011)033. arXiv:1012.4785 [hep-th]

89. N. Evans, C. Miller, M. Scott, Phys. Rev. D 94, 074034 (2016). $\quad$ https://doi.org/10.1103/PhysRevD.94.074034. arXiv:1604.06307 [hep-ph]

90. D. Li, M. Huang, Y. Yang, P.-H. Yuan, JHEP 02, 030 (2017). https://doi.org/10.1007/JHEP02(2017)030. arXiv:1610.04618 [hep-th] 
91. Z. Fang, Y.-L. Wu, L. Zhang, Phys. Lett. B 762, 86 (2016). https:// doi.org/10.1016/j.physletb.2016.09.009. arXiv:1604.02571 [hep$\mathrm{ph}]$

92. E. D'Hoker, P. Kraus, JHEP 03, 095 (2010). https://doi.org/10. 1007/JHEP03(2010)095. arXiv:0911.4518 [hep-th]

93. I.Y. Aref'eva, K. Rannu, P. Slepov, (2020). arXiv:2011.07023 [hep-th]

94. H. Bohra, D. Dudal, A. Hajilou, S. Mahapatra, Phys. Lett. B 801, 135184 (2020). https://doi.org/10.1016/j.physletb.2019.135184. arXiv:1907.01852 [hep-th]

95. U. Gursoy, M. Jarvinen, G. Nijs, Phys. Rev. Lett. 120, 242002 (2018). $\quad$ https://doi.org/10.1103/PhysRevLett.120.242002. arXiv: 1707.00872 [hep-th]

96. D.T. Son, A.O. Starinets, JHEP 09, 042 (2002). https://doi.org/ 10.1088/1126-6708/2002/09/042. arXiv:hep-th/0205051

97. G. Policastro, D.T. Son, A.O. Starinets, JHEP 09, 043 (2002). https://doi.org/10.1088/1126-6708/2002/09/043. arXiv:hep-th/0205052

98. O. DeWolfe, S.S. Gubser, C. Rosen, Phys. Rev. D 83, 086005 (2011). https://doi.org/10.1103/PhysRevD.83.086005. arXiv:1012.1864 [hep-th]
99. R. Rougemont, A. Ficnar, S. Finazzo, J. Noronha, JHEP 04, 102 (2016). https://doi.org/10.1007/JHEP04(2016)102. arXiv:1507.06556 [hep-th]

100. R. Critelli, J. Noronha, J. Noronha-Hostler, I. Portillo, C. Ratti, R. Rougemont, Phys. Rev. D 96, 096026 (2017). https://doi.org/ 10.1103/PhysRevD.96.096026. arXiv:1706.00455 [nucl-th]

101. R. Rougemont, R. Critelli, J. Noronha-Hostler, J. Noronha, C. Ratti, Phys. Rev. D 96, 014032 (2017). https://doi.org/10.1103/ PhysRevD.96.014032. arXiv:1704.05558 [hep-ph]

102. R. Critelli, R. Rougemont, J. Noronha, Phys. Rev. D 99, 066004 (2019). https://doi.org/10.1103/PhysRevD.99.066004. arXiv:1805.00882 [hep-th]

103. R. Rougemont, R. Critelli, J. Noronha, Phys. Rev. D 98, 034028 (2018). https://doi.org/10.1103/PhysRevD.98.034028. arXiv: 1804.00189 [hep-ph]

104. X. Chen, D. Li, D. Hou, M. Huang, JHEP 03, 073 (2020). https:// doi.org/10.1007/JHEP03(2020)073. arXiv:1908.02000 [hep-ph]

105. Z. Fang, Y.-L. Wu, Chin. Phys. C 44, 103101 (2020). https://doi. org/10.1088/1674-1137/abab90. arXiv:1909.06917 [hep-ph] 